\title{
The Role of the Telovelar Approach in Fourth Ventricular Surgery: A New Perspective
}

\author{
Ehab Mohamed EISSA \\ Cairo University, Kasr Al-Aini Medical School, Department of Neurosurgery, Cairo, Egypt
}

\section{ABSTRACT}

AIM: To evaluate the efficiency of the telovelar approach for fourth ventricular lesions through identifying and preserving neurovascular structures.

MATERIAL and METHODS: A total of 40 patients with fourth ventricular tumors were treated via the telovelar approach between 2005 and 2014.

RESULTS: The telovelar approach provided adequate exposure in all patients. The brainstem and posterior inferior cerebellar artery (PICA) were identified early and preserved in all patients. Potential tumor attachment was observed at the floor of the fourth ventricle in $22(55 \%)$ patients: five had brainstem glioma, while 16 of the remaining $17(94 \%)$ had focal attachments at any area of the caudal fourth ventricular floor, and two (11.7\%) had attachments at any area of the lateral aspect of the rostral fourth ventricular floor, which was the only point of attachment in one. None of these tumors infiltrated the area of the cerebral aqueduct. Gross total excision was achieved in $45 \%$ of patients and near total excision was possible in $25 \%$ due to focal tumor attachment at one or more of the previously mentioned areas. However, debulking was possible in $30 \%$.

CONCLUSION: The telovelar approach provides the advantages of early identification and preservation of the brainstem and PICA, and allows for assessment of potential tumor attachment at the aforementioned areas. This is in contrast to the traditional consideration of vermian preservation, which is impossible to achieve regardless of the approach in patients with large tumors infiltrating the vermis. The pathological nature of the tumor and the degree of brainstem infiltration were key factors that determined the degree of tumor excision.

KEYWORDS: Children, Fourth ventricular surgery, Posterior fossa tumor, Telovelar approach

\section{INTRODUCTION}

$\mathrm{F}$ Tourth ventricular lesions present a neurosurgical challenge not only because of their deep location, relationship with important neurovascular structures, and high morbidity and mortality rates, but also due to the frequency of tumors in this region, especially in children $(6,8,18,24)$. Traditionally, the fourth ventricular region has been accessed via the transvermian approach described by Dandy in 1966 after splitting the inferior vermis $(3,10,11)$. However, many morbidities have been reported with this technique, such as balance disturbances and cerebellar mutism $(4,22,23)$.
Advancements in micro neurosurgical anatomy and surgical techniques have led neurosurgeons to search for natural corridors for accessing this deep cranial region, because proper dissection along the natural avascular planes will preserve important structures and minimize complications. In 1992, Matsushima et al. reported on the use of the cerebellomedullary fissure to access this region (14). With good results achieved, this avenue has gained popularity among neurosurgeons $(7,9,12-16,18-20,25,26)$. Most studies describing the telovelar approach have focused on its advantage in vermian preservation $(15,18,21,23)$, and neglected the importance of a 
natural corridor approach in the early identification of the relationship between the tumor and the neurovascular structures.

The aim of this study is to evaluate the efficiency and safety of the telovelar approach for removing fourth ventricular lesions through identifying and preserving important neurovascular structures.

\section{MATERIAL and METHODS}

This retrospective study included 40 children with fourth ventricular tumors treated via the telovelar approach between 2005 and 2014. Each case was analyzed according to sex, age, clinical presentation, preoperative computed tomography (CT), magnetic resonance imaging (MRI), pathology, surgical intervention, and outcomes. The patients were followed at our outpatient clinic, where they were assessed clinically by their history and physical examination, functionally by the Karnofsky performance scale, and radiologically by CT and/ or MRI of the brain.

\section{Operative Technique}

Cooperation with the anesthesia team was necessary to detect any complications related to positioning, such as an air embolism, or to brainstem manipulation during tumor removal, such as hypertension and bradycardia.

All patients were placed in the prone position to avoid the risk of air embolism complications, which have been reported with the sitting position. This position also facilitated the angle of exposure provided by the telovelar approach. The head was flexed to a distance approximately two fingerbreadths between the chin and neck to avoid intraoperative congestion and increased intracranial tension. Exposure was performed via a suboccipital craniotomy or craniectomy with removal of the posterior arch of the atlas to assist in visualizing the lower pole of the cerebellar tonsils, which was displaced downward in some patients. The dura was opened in a Y-shaped fashion and the arachnoids covering the cisterna magna were incised to release cerebrospinal fluid (CSF) as well as to identify the lower pole of both tonsils and the cerebellomedullary fissure (Figure 1A-D).

The cerebellomedullary fissure was opened as described previously $(7,9,12-16,18)$. The fissure is composed of two spaces, the tonsillouvular and tonsillomedullary spaces. The arachnoids between the tonsils, the tonsils and medulla, and the tonsils and uvula were dissected completely to allow for free retraction of the tonsils and uvula. Then, the lower part of the fourth ventricular roof was exposed, including the tela choroidea and inferior medullary velum, which usually were stretched by the tumor and could not be identified as separate structures; this layer was incised.

With large tumors, as was usually the case in our study, the lower pole of the tumor can be clearly visualized after opening the fissure or even after opening the cisterna magna (Figure 2).

The relationship between the tumor and brainstem was identified by inspecting and manipulating the lower pole of the tumor, which revealed one of the following two situations:

The lower pole of the tumor was not attached to the obex and so it was elevated, exposing the brainstem with its white glistening appearance. Then, a cottonoid was placed under the tumor over the floor of the fourth ventricle. The operation proceeded by debulking the tumor at this stage because nonattachment of the lower pole of the tumor to the obex does not exclude another point of attachment. After debulking, the remaining small part was easily inspected for removal or left according to its relationship with the brainstem (Figure 3).

The lower pole of the tumor was attached to the obex and debulking proceeded, which was guided by an imaginary line directed from the obex rostrally and downward toward the
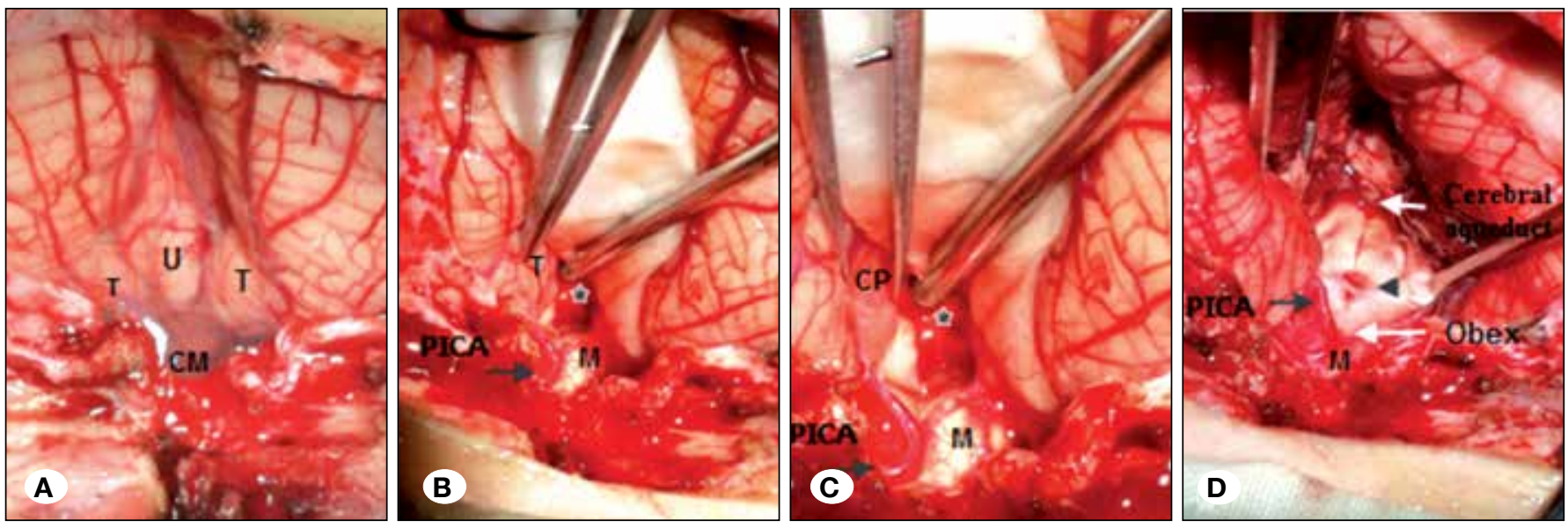

Figure 1: Intraoperative views of a telovelar approach to a $4^{\text {th }}$ ventricular tumor.

A) After dural opening and removal of the C1 posterior arch and exposure of cistern magna; B) after opening the arachnoids of the cisterna magna, beginning of the opening of the cerebellomedullary fissure with exposure of spino-medullary junction, left PICA, obex and the tumor that just reaching the obex; C) after opening of the fissure with early identification of relation between the tumor and neurovascular structures; D) the final view after complete tumor excision. (T: Tonsil, U: Uvula, CM: Cerebellomedullary fissure, M: Medulla, PICA: Posterior inferior cerebellar artery, *: Tumor, CP: Cerebellar peduncle). 


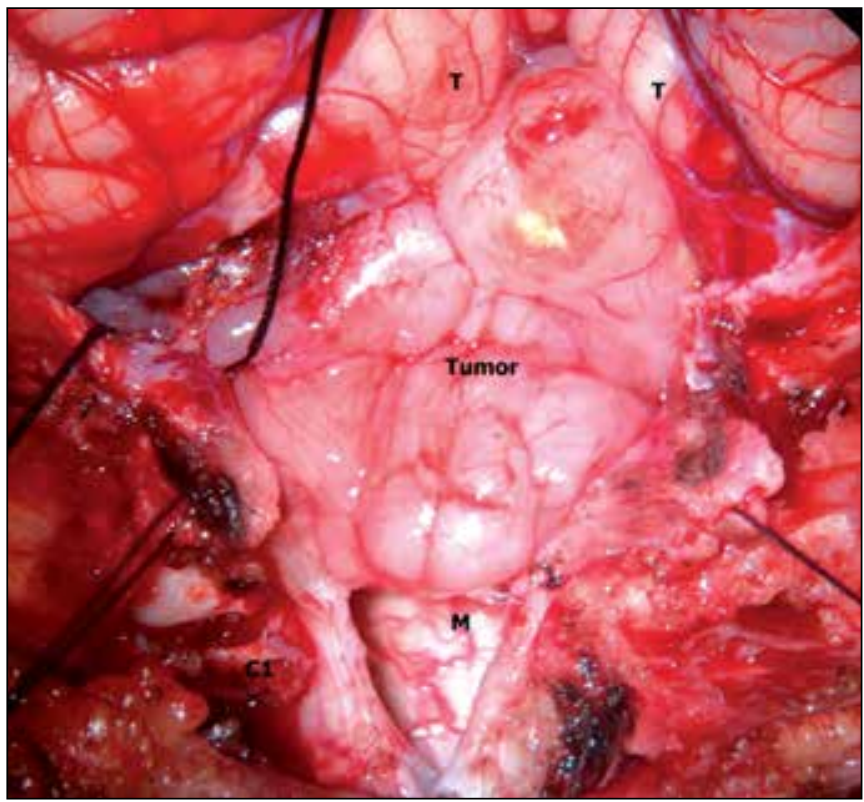

Figure 2: Operative view of fourth ventricular tumor extending beyond the obex with early identification of neurovascular structures through the telovelar approach.

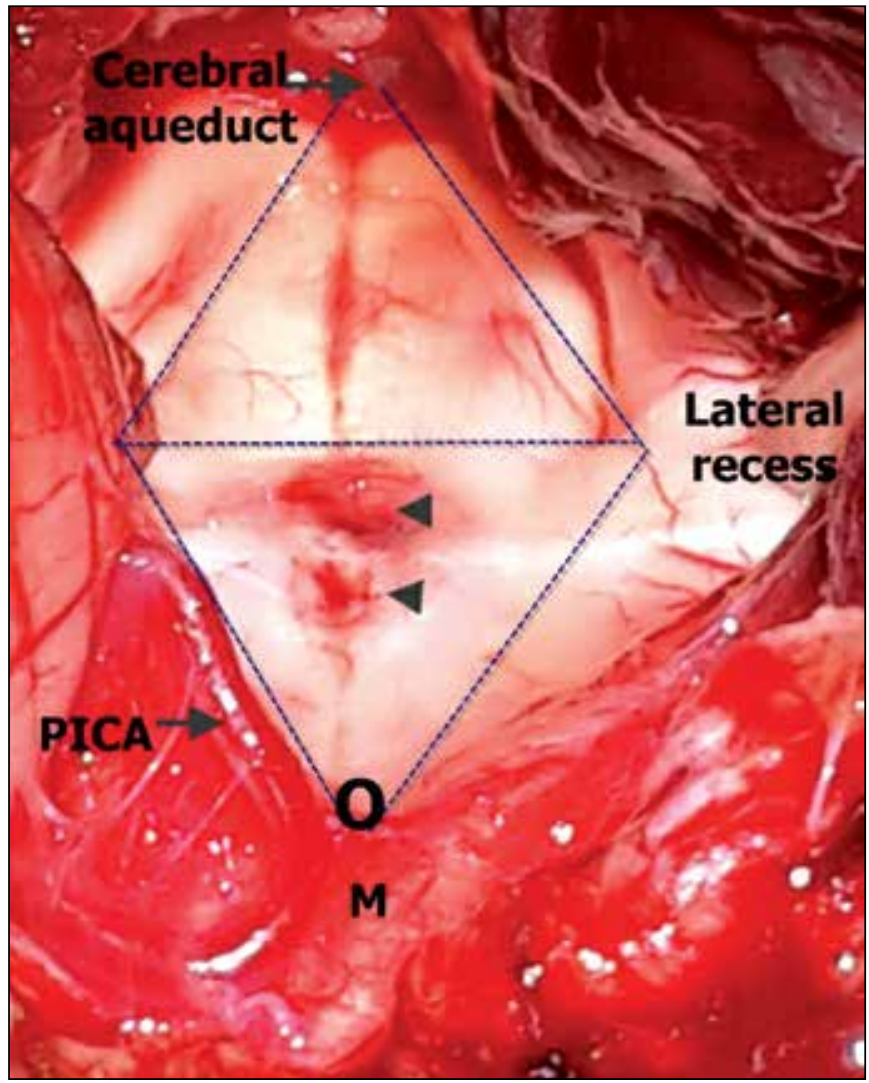

Figure 3: Another intraoperative microscopic view showing the exposure of the entire $4^{\text {th }}$ ventricular floor up to the aqueduct of Sylvius via the telovelar approach with the lower triangle representing the most common points of potential tumor attachment drawn at the caudal half from the obex inferiorly and the level of the lateral recesses bilaterally. direction of the aqueduct of Sylvius, which corresponded to the floor of the fourth ventricle. After debulking, a thin carpet was left covering the brainstem and further management proceeded according to the degree of tumor attachment to the fourth ventricular floor, depending on whether there was only focal attachment at the obex, additional points, or the entire floor of the fourth ventricle.

The posterior inferior cerebellar artery (PICA), usually the telovelotonsillar segment, could be identified early after opening the fissure. The PICA was dissected from the tumor at the beginning of surgery by cutting the arachnoidal adhesion or by shaving part of the tumor attached to the PICA and leaving this part until the end of the operation.

\section{Postoperative Management}

The patient was placed in the Intensive Care Unit for at least one day and was extubated after complete recovery of consciousness. The anesthetist was instructed to examine the mobility of the vocal cord to exclude lower cranial nerve palsy. Oral feeding was postponed until postoperative day 2. Under supervision of the neurosurgery resident, a small amount was given using a teaspoon after exclusion of bulbar manifestations, which were suggested by voice changes, inability to swallow saliva, and absence of a cough reflex.

\section{RESULTS}

We studied 40 patients with fourth ventricular tumors (average age at presentation, $7.3 \pm 1.4$ years; range, 10 months-12 years). There was a slight male predominance, with a maleto-female ratio of 22/18 (1.2). The most common presentation was intracranial hypertension (vomiting, headache, and sixth nerve palsy) in $90 \%$ of the patients followed by ataxia (35\%), eye manifestations (diminution of vision due to long-standing papilledema in $20 \%$ ), and bulbar manifestations (10\%).

The exposure provided by this approach was adequate in all patients after routine removal of the posterior arch of $\mathrm{C} 1$, even if significant downward displacement of the tonsils was present, which is not uncommon in such patients. In fact, downward displacement of the tonsils below the foramen magnum was noted in 6 patients (15\%). It is noteworthy that the narrow working angle toward the upper part of the floor of the fourth ventricle was efficiently compensated by changing the angle and direction of the microscope and table.

All procedures were performed without a vermian incision, except in three patients (two with medulloblastomas and one with astrocytoma) aged 10 months, and 1.5 and 3 years. The tumors were very vascular in these patients; therefore, the vermis was incised to increase the working angle and achieve rapid tumor removal because prolongation of the operative time was expected to endanger the patient's life.

\section{Potential Site of Tumor Attachment}

Tumor attachment was observed at the floor of the fourth ventricle in $22(55 \%)$ patients. Among these 22 patients, five had brainstem tumors and 16 of the remaining 17 (94\%) had attachment at any area of the caudal fourth ventricular floor, 
representing an inverted triangle with the obex inferiorly and the level of lateral recesses bilaterally (Figure 3). Two tumors $(11.7 \%)$ were attached at any area of the lateral aspect of the rostral fourth ventricular floor, which was the only point of attachment in one of them. None of these tumors infiltrated the area of the cerebral aqueduct.

Gross total removal was achieved in 18 patients (45\%), where there was no attachment to the floor of the fourth ventricle. Near total (leaving $<1.5 \mathrm{~cm}^{2}$ ) removal was possible in 10 patients (25\%) due to focal tumor attachment at one or more of the previously mentioned areas (case presentation). Both groups represented $70 \%$ of the patients. However, in 12 patients (30\%), only debulking was possible because the tumor either originated from the brainstem (five brainstem gliomas) or was attached extensively to the floor (six ependymomas and one medulloblastoma; Figures 4A-D and 5A-D).

The most common tumors were medulloblastomas $(52.5 \%)$, followed by astrocytomas $(22.5 \%)$, and ependymomas (17.5\%). The less common types were hemangioblastomas and dermoid and choroid plexus papillomas (one case each $=2.5 \%)$.

\section{Complications and Outcomes}

There were no intraoperative or immediate postoperative mortalities, and no PICA, brainstem injury, or postoperative bulbar manifestations. The mean preoperative Karnofsky performance scale was 82 (range, 40-100), which improved postoperatively to 90 (range, 60-100). Of 14 patients who presented preoperatively with significant ataxia, the condition remained the same in 10, worsened in three, and improved in one. Additionally, four new patients suffered ataxia: three cases resolved within 3 months and one patient suffered mutism that resolved within 2 months. It is noteworthy that this patient was treated without vermian splitting.

\section{DISCUSSION}

The transvermian approach described by Dandy (3) to treat fourth ventricular tumors through the inferior vermian incision has fallen out of favor in the last 20 years due to the significant approach-related morbidity (caudal vermis syndrome) in the form of equilibrator disturbance (23). Furthermore, there is a risk of damage to the dentate nucleus, which is in relation to the posterolateral margin of the fourth ventricular roof rostral to the superior pole of the tonsil (1), with subsequent, severe equilibrator disturbances $(5,15,18,20)$. Additionally, vermian splitting can result in cerebellar mutism, which is a transient complication characterized by a lack of speech in the awake patient, with intact speech comprehension that sometimes is
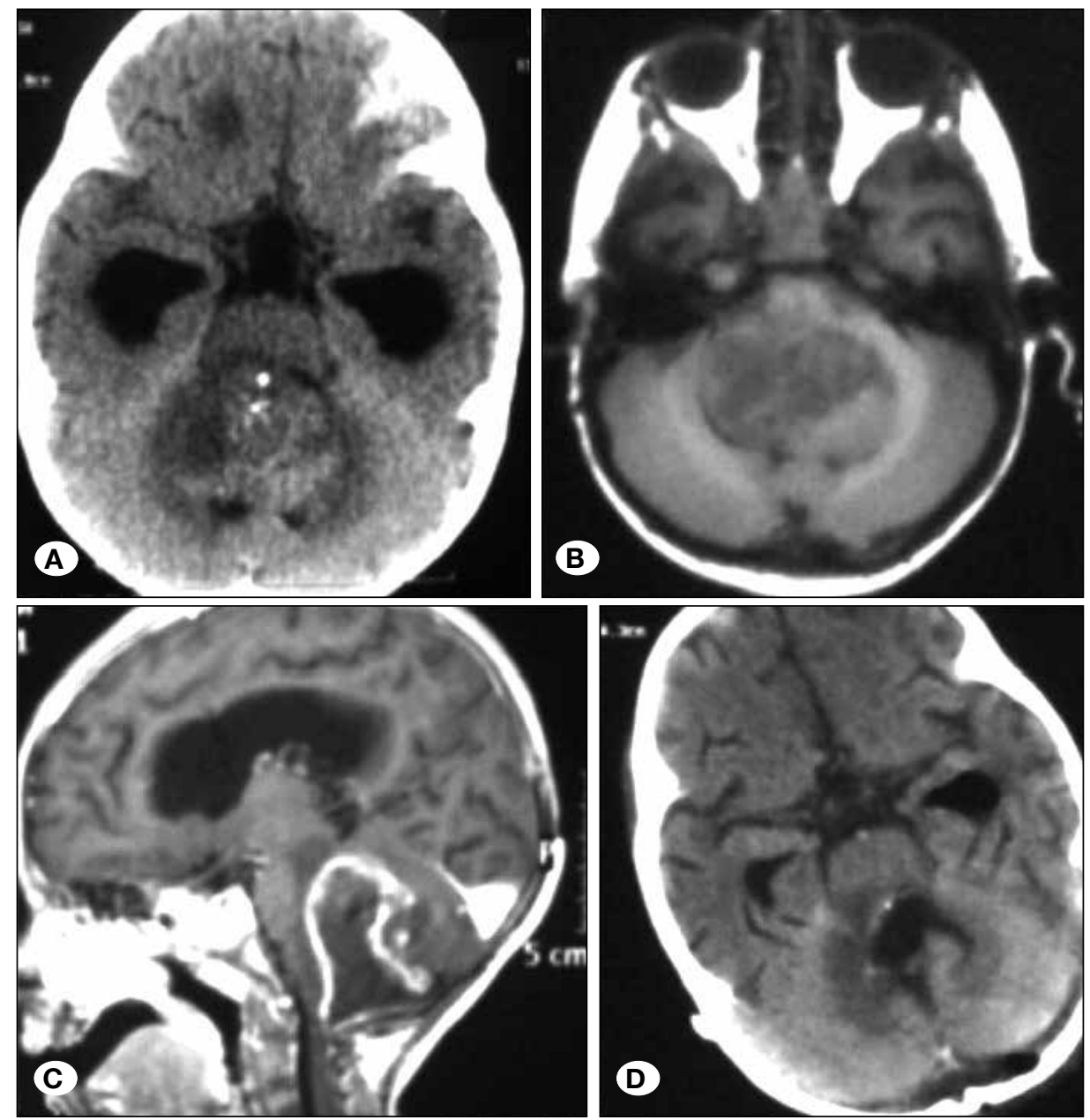

Figure 4: Patient with ependymoma attached focally at the obex excised near totally. A) Preoperative axial plain CT scan, B) preoperative axial MRI, C) preoperative sagittal MRI with contrast showing focal attachment to the obex, D) postoperative CT scan showing near total tumor excision without vermian splitting. 
associated with oral apraxia $(4,22)$. Additionally, this approach did not provide proper exposure of the lateral aspects of the fourth ventricular floor (4).

Matsushima et al. recommended approaching the fourth ventricle by opening the natural cleft (cerebellomedullary fissure) in 1992, simulating the Sylvian fissure opening in the pterional approach (14). Many studies followed describing this corridor $(4,18-20,25,26)$, especially the study of Rhoton $(18,20)$.

The cerebellomedullary fissure is related anteriorly to the posterior surface of the medulla, superiorly to the inferior medullary velum and tela choroidea, posteriorly to the uvula in the midline, and laterally to the tonsils and biventral lobules. It communicates around the tonsils with the cisterna magna, through the lateral recess and foramen of Luschka with the common pericallosal arteries, and through the foramen of Magendie with the fourth ventricle; therefore, one can imagine the total area provided by this cleft $(18,20)$.

Operative details about this approach vary widely among studies dealing with cadaveric dissection or including small tumors to those dealing with large size tumors $(5,19)$. In many reports, the anatomical structures were identified clearly and the microsurgical dissection proceeded after freeing the tonsils and uvula by cutting the taenia choroidea and the posterior margin of the lateral recess, which was described by Matsushima as the extensive opening method when performed bilaterally and as the lateral wall opening method when performed on one side (14-17). Kellogg and Piatt also described opening of the fissure in the same way (10), but they used a simpler expression in the form of an incision extending from the foramen of Magendie to the foramen of Luschka through the tela choroidea. Rhoton usually added an incision to the inferior medullary velum for optimal exposure of the floor $(18,20)$.

In the current study, large fourth ventricular tumors usually were encountered, which was the same problem as in other developing countries $(5,19)$ due to late diagnosis. These large tumors were associated with hydrocephalus, which was reported in all of our patients. The routine protocol for hydrocephalus was preoperative CSF diversion, which was performed before tumor surgery but in the last patients of our study, CSF diversion and tumor resection were performed in the same setting to avoid the risk of upward herniation. Preoperative CSF diversion is very helpful in such patients as it facilitates intraoperative dissection and retraction by lowering
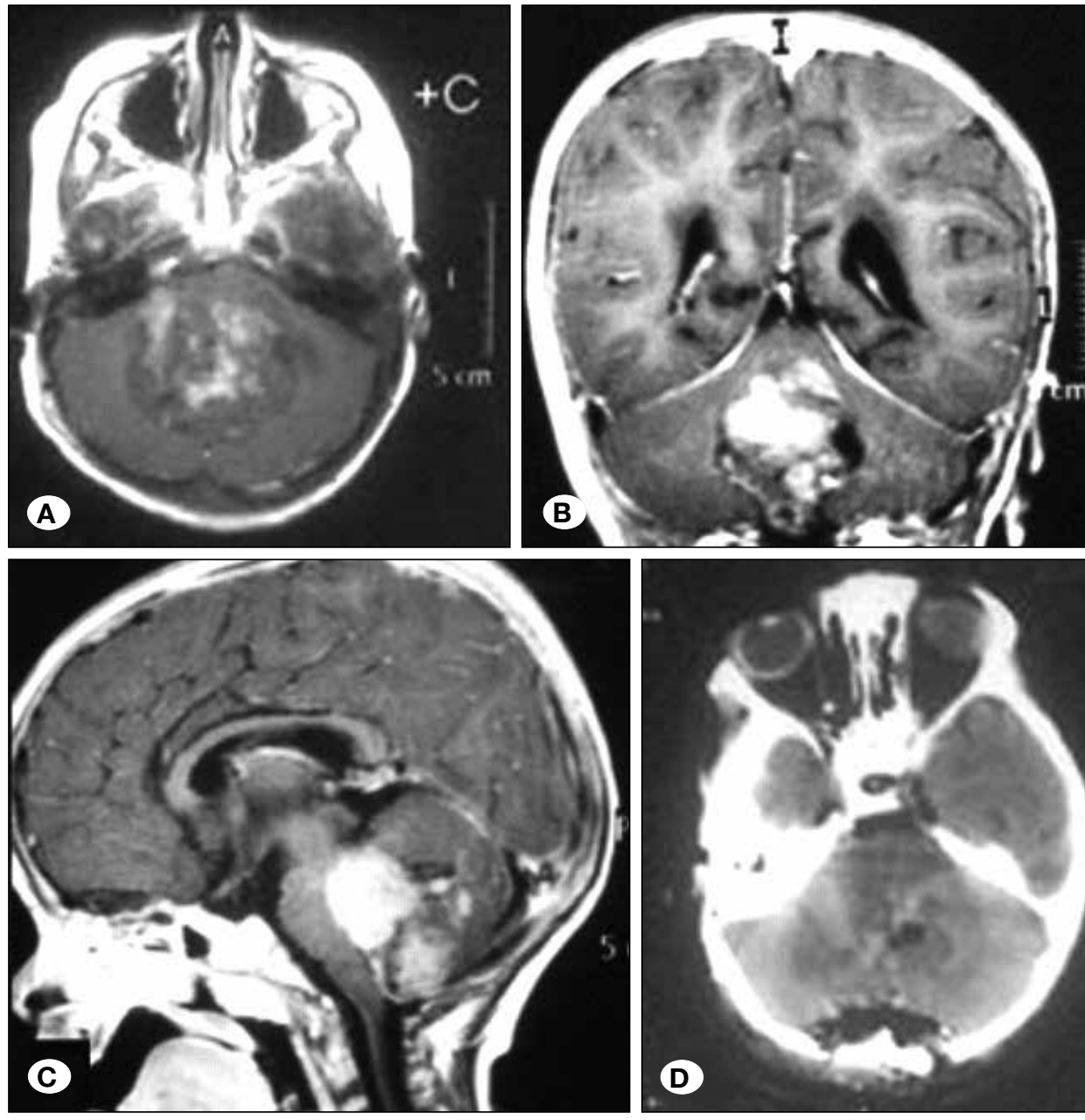

Figure 5: Patient with medulloblastoma not attached to brainstem, but attached to middle cerebellar peduncles excised near totally.

A) Preoperative axial, B) preoperative coronal and C) preoperative sagittal brain MRI with contrast.

D) Postoperative CT scan showing near total tumor excision without vermian splitting. 
intracranial tension. In addition, it protects against sudden or late postoperative hydrocephalus, which is not uncommon after fourth ventricular surgery. In addition, these large tumors distorted the normal anatomy and extended laterally to the cerebellopontine angles, inferiorly out of the foramen of Magendie and/or superiorly towards the posterior third ventricle and, therefore, the lower part of the fourth ventricular roof, including the tela choroidea and inferior medullary velum, was usually stretched by the tumor capsule and could not even be identified as separate structures in most patients.

After opening the fissure, the lower pole of the tumor could be inspected and manipulated to identify its relationship with the brainstem (as mentioned before) and PICA, which is usually encountered between the tonsil on one side and the tela and velum on the opposite side (telovelotonsillar segment) $(20,24)$. At this stage, the operation proceeded by debulking the tumor without dissection guided by the orientation provided by this approach regarding neurovascular structures. Consistent with other studies describing the removal of large tumors via this approach (19), we found that dissection of this natural cleft before decompression may damage the vital structures related to this region. Decompression and dissection should be performed simultaneously, leaving only a small part or carpet of the tumor that can be removed in the final step of the operation according to its degree of attachment.

Our study provided special data with respect to the potential sites of tumor attachment to the brainstem. The lower part of the floor of the fourth ventricle (which corresponds to an inverted triangle with the apex at the obex and the sides related to the lateral aspect of the floor and inferior cerebellar peduncles at the level of lateral recesses bilaterally) was the most common site of any area of attachment in our study, and no patient had infiltration of the upper part of the floor around the aqueduct of Sylvius. This triangle is related to an important area of the fourth ventricular floor, which includes:

1. Medially from above the downward facial colliculus, a safe area of 3 to $4 \mathrm{~mm}$ in length, the hypoglossal and vagal areas, and the area postrema,

\section{Laterally, the vestibular area.}

The upper part of the fourth ventricular floor is considered anatomically as a safe area during the surgical approaches that require going through it. The exact dimension of these areas was provided in the pioneering work of Bogucki et al. (2). Now, the major importance of the telovelar approach provided in this study is that it enables the surgeon to identify early the most dangerous part of the tumor (the part with the most common incidence of attachment and the part related to the most important brainstem nuclei) and treat it appropriately. In addition, it provides early identification of the vascular structures and their relationship with the tumor.

Pathological data support our operative findings of tumor attachment at the caudal brainstem. Medulloblastomas, which represent the majority of fourth ventricular tumors, originate mostly from the inferior medullary velum, while ependymomas arise most commonly from the lower half of the floor of the fourth ventricle (8).
The most commonly reported limitation of the telovelar approach is the narrow working angle toward the upper half of the fourth ventricle (23). This misconception can be addressed by changing the angle of the microscope and operating table. Moreover, surgical dissection at the rostral part of the fourth ventricle is relatively safer, as mentioned previously (2), and easier due to the lack of tumor attachment compared to the caudal part. Deshmukh et al. reported that removal of the C1 posterior arch increased the working angle toward the upper half of the fourth ventricle (4), which was equivalent to that provided by transvermian access. This step was performed routinely in our study not only due to the mentioned reason but also because it was essential to open the cerebellomedullary fissure in patients with downward displacement of the tonsils (secondary Chiari malformation), which was documented in $15 \%$ of our patients.

A combined approach with a vermian incision was needed in only three of our patients with very vascular tumors at a young age to increase the working angle for rapid tumor removal without more blood loss, which was expected to endanger the life of the patient. It should be noted that opening of the vermis was inevitable after removal of large tumors infiltrating the vermis.

In this study, total tumor excision was achieved in 18 patients (45\%), near total removal (leaving less than $1.5 \mathrm{~cm}$ ) in 10 (25\%), and subtotal removal in $15(30 \%)$. These results were not related to inadequate exposure via this approach, which was sufficient in all patients. Instead, they were due to the nature of the tumor and the degree of brainstem infiltration. Any attempt to remove parts of a tumor that infiltrated the brainstem or was tightly adhering to the cranial nerves was avoided to prevent postoperative morbidity, especially a bulbar symptom $(2,15)$. All patients recovered uneventfully postoperatively without bulbar manifestations.

Four patients had postoperative ataxia, which resolved within 3 months in three of them. Additionally, one child with medulloblastoma suffered cerebellar mutism that resolved within 3 months. It is noteworthy that this patient had not undergone a vermian incision. Therefore, cerebellar complications can occur, albeit less commonly with the telovelar approach than with the transvermian approach. Such cerebellar complications may be related to the infiltrative nature of tumors to the vermis rather than the surgical experience or the approach (5).

\section{CONCLUSION}

The telovelar approach has the advantage of early identification and preservation of the brainstem and PICA. In addition, it allows for the assessment of potential tumor attachment at the aforementioned areas rather than the traditional consideration of vermian preservation, which is impossible to achieve in patients with large tumors infiltrating the vermis regardless of the approach. The pathological nature of the tumor and the degree of brainstem infiltration are key factors that determine the degree of tumor excision. 


\section{REFERENCES}

1. Akakin A, Peris-Celda M, Kilic T, Seker A, Gutierrez-Martin A, Rhoton A Jr: The dentate nucleus and its projection system in the human cerebellum: The dentate nucleus microsurgical anatomical study. Neurosurgery 74(4):401-424; discussion 424-425, 2014

2. Bogucki J, Gielecki J, Czernicki Z: The anatomical aspects of a surgical approach through the floor of the fourth ventricle. Acta Neurochir (Wien) 139(11):1014-1019, 1997

3. Dandy WE: The Brain. In: Lewis D (ed), Practice of Surgery: Hagerstown, MD: WF Prior, 1966:452-458

4. Deshmukh VR, Figueiredo EG, Deshmukh P, Crawford NR, Preul MC, Spetzler RF: Quantification and comparison of telovelar and transvermian approaches to the fourth ventricle. Neurosurgery 58(4 Suppl 2): ONS-202-206; discussion ONS206-207, 2006

5. El-Bahy K: Telovelar approach to the fourth ventricle: Operative findings and results in 16 cases. Acta Neurochir 147(2):137142, 2005

6. Gok A, Alptekin M, Erkutlu I: Surgical approach to the fourth ventricle cavity through the cerebellomedullary fissure. Neurosurg Rev 27(1): 50-54, 2004

7. Jean WC, Abdel Aziz KM, Keller JT, van Loveren HR: Subtonsillar approach to the foramen of Luschka: An anatomic and clinical study. Neurosurgery 52(4):860-866; discussion 866, 2003

8. Jung TY, Rutka JT: Posterior fossa tumors in the pediatric population. Multidisciplinary management. In: Hinojosa AQ (ed), Schmidek and Sweet: Operative Neurosurgical Techniques, Indications, Methods and Results. $6^{\text {th }}$ ed. Saunders, 2012:654-668

9. Kawashima M, Matsushima T, Nakahara Y, Takase Y, Masuoka J, Ohata K: Trans-cerebellomedullary fissure approach with special reference to lateral route. Neurosurg Rev 32(4):457464, 2009

10. Kellogg JX, Piatt JH Jr: Resection of fourth ventricle tumors without splitting the vermis: The cerebellomedullary fissure approach. Pediatr Neurosurg 27:28-33, 1997

11. Kempe LG: Operative neurosurgery. New York: SpringerVerlag, 1970:1-3

12. Lee CC1, Lin CF, Yang TF, Hsu SP, Chen HH, Chen SC, Shih $\mathrm{YH}$ : Telovelar approach for choroid plexus papilloma in the foramen of Luschka: A safe way using a neuromonitor. Clin Neurol Neurosurg 114(3): 249-253, 2012
13. Liu R, Kasper EM: Bilateral telovelar approach: A safe route revisited for resections of various large fourth ventricle tumors. Surg Neurol Int 30:5-16, 2014

14. Matsushima T, Fukui $M$, Inoue $T$, Natori $Y$, Baba T, Fujii $K$ : Microsurgical and magnetic resonance imaging anatomy of the cerebello-medullary fissure and its application during fourth ventricle surgery. Neurosurgery 30(3):325-330, 1992

15. Matsushima T, Inoue T, Inamura T, Natori Y, Ikezaki K, Fukui M: Transcerebellomedullary fissure approach with special reference to methods of dissecting the fissure. J Neurosurg 94(2): 257-264, 2001

16. Matsushima T, Rhoton AL Jr, Lenkey C: Microsurgery of the fourth ventricle: Part 1. Microsurgical anatomy. Neurosurgery 11(5): 631-667, 1982

17. Matsushima T, Abe H, Kawashima M, Inoue T: Exposure of the wide interior of the fourth ventricle without splitting the vermis: Importance of cutting procedures for the tela choroidea. Neurosurg Rev 35(4):563-571; discussion 571-572, 2012

18. Mussi AC, Rhoton AL Jr: Telovelar approach to the fourth ventricle: Microsurgical anatomy. J Neurosurg 92(5):812-823, 2000

19. Rajesh BJ, Rao BR, Menon G, Abraham M, Easwer HV, Nair S: Telovelar approach: Technical issues for large fourth ventricle tumors. Childs Nerv Syst 23(5):555-558, 2007

20. Rhoton AL Jr: Cerebellum and fourth ventricle. Neurosurgery 47 Suppl 3: S7-27, 2000

21. Shimoji K, Miyajima M, Karagiozov K, Yatomi K, Matsushima $\mathrm{T}$, Arai $\mathrm{H}$ : Surgical considerations in fourth ventricular ependymoma with the transcerebellomedullary fissure approach in focus. Childs Nerv Syst 25(10):1221-1228, 2009

22. Steinbok P, Cochrane DD, Perrin R, Price A: Mutism after posterior fossa tumour resection in children: Incomplete recovery on long-term follow-up. Pediatr Neurosurg 39(4): 179-183, 2003

23. Tanriover N, Ulm AJ, Rhoton AL Jr, Yasuda A: Comparison of the transvermian and telovelar approaches to the fourth ventricle. J Neurosurg 101(3):484-498, 2004

24. Ucerler H, Saylam C, Cagli S, Orhan M, Zileli M: The posterior inferior cerebellar artery and its branches in relation to the cerebellomedullary fissure. Clin Anat 21(2):119-126, 2008

25. Zaheer SN, Wood M: Experiences with the telovelar approach to fourth ventricular tumors in children. Pediatr Neurosurg 46(5):340-343, 2010

26. Ziyal IM, Sekhar LN, Salas E: Subtonsillar-transcerebellomedullary approach to lesions involving the fourth ventricle, the cerebellomedullary fissure and the lateral brainstem. $\mathrm{Br} J$ Neurosurg 13(3):276-284, 1999 